\title{
SCIENTIFIC REP

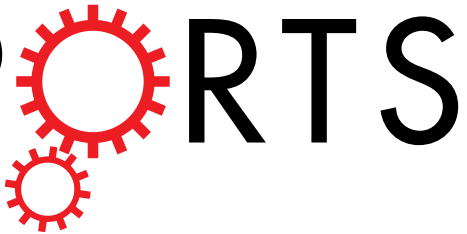 \\ OPEN From cholera to corals: Viruses as drivers of virulence in a major coral bacterial pathogen
}

Received: 17 June 2015

Accepted: 09 November 2015

Published: 08 December 2015

\author{
Karen D. Weynberg ${ }^{1}$, Christian R. Voolstra ${ }^{2}$, Matthew J. Neave ${ }^{2}$, Patrick Buerger ${ }^{1,3,4}$ \& \\ Madeleine J. H. van Oppen ${ }^{1,5}$
}

Disease is an increasing threat to reef-building corals. One of the few identified pathogens of coral disease is the bacterium Vibrio coralliilyticus. In Vibrio cholerae, infection by a bacterial virus (bacteriophage) results in the conversion of non-pathogenic strains to pathogenic strains and this can lead to cholera pandemics. Pathogenicity islands encoded in the $V$. cholerae genome play an important role in pathogenesis. Here we analyse five whole genome sequences of $V$. coralliilyticus to examine whether virulence is similarly driven by horizontally acquired elements. We demonstrate that bacteriophage genomes encoding toxin genes with homology to those found in pathogenic $V$. cholerae are integrated in $V$. coralliilyticus genomes. Virulence factors located on chromosomal pathogenicity islands also exist in some strains of $V$. coralliilyticus. The presence of these genetic signatures indicates virulence in $V$. coralliilyticus is driven by prophages and other horizontally acquired elements. Screening for pathogens of coral disease should target conserved regions in these elements.

Species of the gammaproteobacterium Vibrio are well-known for their roles as pathogens in both terrestrial and aquatic environments ${ }^{1}$. However, not all species and strains of Vibrio are pathogenic ${ }^{2,3}$. For instance, Vibrio cholerae, a bacterium that causes the acute diarrhoeal disease cholera ${ }^{4-6}$ requires the $^{-}$ acquisition of key virulence factors to become toxigenic. The two major virulence factors of $V$. cholerae, TCP (toxin co-regulated pilus) and an exotoxin called cholera toxin (CTX), are both introduced via horizontal gene transfer (HGT) and are encoded by a pathogenicity island ${ }^{7}$ and the genome of a single-stranded DNA lysogenic filamentous bacteriophage (or prophage) called CTXphi $(\mathrm{CTX} \varphi)^{6}$, respectively. The TCP receptor is a prophage receptor facilitating introduction of CTX $\varphi$ prophage DNA into the cell ${ }^{6}$. In $V$. cholerae, $\mathrm{CTX} \varphi$ prophage transmits toxin genes among the bacteria that express the TCP prophage receptor. This process of inducing virulence in an otherwise harmless bacterium through prophage infection and integration of the prophage genome into the bacterial host genome is called lysogenic conversion ${ }^{8}$.

The health of coral reefs is declining on a global scale ${ }^{9,10}$. One of the causes of this deterioration is coral disease, the incidence and severity of which has increased worldwide over recent decades ${ }^{11-13}$. A number of coral disease studies have reported Vibrio species, V. coralliilyticus in particular, as the pathogen responsible for some instances of white syndrome (WS) disease ${ }^{14,15}$, as well as coral bleaching ${ }^{16,17}$. Coral bleaching is the loss of the algal endosymbiont, Symbiodinium, and/or its photosynthetic pigments from the coral tissue resulting in paling of the coral tissues. If these coral algal endosymbionts are not regained following bleaching, coral mortality is inevitable ${ }^{18}$. Bleaching tends to occur more gradually 


\begin{tabular}{|c|c|c|c|c|c|c|}
\hline $\begin{array}{l}\text { Vibrio coralliilyticus } \\
\text { strain }\end{array}$ & $\begin{array}{l}\text { Geographical origin } \\
\text { of isolation }\end{array}$ & Species strain isolated from & Genome Accession No. & $\begin{array}{l}\text { Genome } \\
\text { size }(\mathrm{Mb})\end{array}$ & Disease state & Reference \\
\hline BAA450 & $\begin{array}{l}\text { Zanzibar, Tanzania } \\
\text { (ATCC, USA) }\end{array}$ & Pocillopora damicornis & ACZN00000000.1 & 5.68063 & Bleaching/White syndrome & Kimes et al., $2012^{20}$ \\
\hline $\mathrm{P} 1$ & $\begin{array}{l}\text { Nelly Bay, Magnetic } \\
\text { Isld, Great Barrier } \\
\text { Reef, Australia }\end{array}$ & Montipora aequituberculata & AEQS00000000.1 & 5.51326 & White syndrome & $\begin{array}{c}\text { Sussman et al., } \\
\text { 2008 }{ }^{15} \text {, Santos et al., } \\
2011^{25}\end{array}$ \\
\hline OCN008 & $\begin{array}{c}\text { Kaneohe Bay, Hawaii, } \\
\text { USA }\end{array}$ & Porites compressa & AVOO00000000.1 & 5.5349 & White syndrome & $\begin{array}{l}\text { Ushijima et al., } \\
2014^{28}\end{array}$ \\
\hline OCN014 & Palmyra Atoll & Acropora cytherea & СР009264; СР009265 & 5.732794 & White syndrome & $\begin{array}{l}\text { Ushijima et al., } \\
2014^{27}\end{array}$ \\
\hline RE98 & Oregon, USA & Pacific oyster Crassostrea gigas & СР009617; СР009618 & 6.03782 & Shellfish pathogen & $\begin{array}{l}\text { Richards et al., } \\
2014^{26}\end{array}$ \\
\hline
\end{tabular}

Table 1. Description of the five whole genome sequences of Vibrio coralliillyticus (four isolated from corals and one from oysters) analysed in this study and implicated in diseases of corals and oysters.

in comparison to the acute disease of WS that results in rapid and irreversible loss of tissue biomass (between 1 to $\left.100 \mathrm{~cm}^{2} \mathrm{day}^{-1}\right)^{19}$. Tissue loss can result in white spots, bands or patches of exposed skeleton with a distinct lesion boundary between exposed skeleton and visibly healthy tissue $e^{17,19}$.

Pathogenicity of the coral-associated V. coralliilyticus in coral disease and bleaching has been attributed to increases in sea surface water temperatures ${ }^{14,20}$, and the immunocompetence of the coral host ${ }^{21}$. However, the underlying mechanisms are not understood, and a possible role for prophages in driving virulence of coral pathogens, as is the case for $V$. cholerae, has not yet been considered. Inconsistent findings on the role of Vibrio in coral disease/bleaching events have been reported in the literature ${ }^{16,22,23}$. In the case of the coral pathogen $V$. coralliilyticus, identified as the causative pathogen of WS in the coral Pocillopora damicornis ${ }^{14,15}$, it was initially observed that $V$. coralliilyticus encodes for a zinc-metalloprotease that causes cellular damage in coral tissue ${ }^{17,24}$. However, a recent study did not detect differences in pathogenicity of a $V$. coralliilyticus strain (P1) between a wild-type and zinc-metalloprotease-deficient mutant in infection experiments ${ }^{25}$, indicating that factors other than zinc-metalloproteases (e.g. hemolysin activity; other proteases) produced by $V$. coralliilyticus strains may also be at play and contribute to virulence. Moreover, the possible role of a prophage as an alternative driver of virulence in $V$. coralliilyticus in these studies has not been addressed.

The aim of this study was to assess whether a prophage could potentially drive virulence of $V$. coralliilyticus by examining in silico five publically available $V$. coralliilyticus whole genome sequences ${ }^{20,25-28}$. Our focus was on the presence of prophage signatures and virulence factors in these genomes, to identify lysogenic conversion by a prophage. Our analysis demonstrates the presence of lysogenic prophages in $V$. coralliilyticus genomes that share similarities to prophages integrated in pathogenic $V$. cholerae strain genomes and supports our hypothesis.

\section{Results}

Recent studies have reported five whole genome sequences of $V$. coralliilyticus associated with corals found in different locations worldwide (Table 1). The first strain of $V$. coralliilyticus to be sequenced was isolated from diseased and bleached corals off the coast of Zanzibar ( V. coralliilyticus ATCC-BAA-450 ${ }^{14,20}$ referred to here as BAA450). Subsequently, whole genome sequences were published for a strain isolated from a Great Barrier Reef coral (V. coralliilyticus $\mathrm{P} 1^{15,25}$ ), and two strains isolated from corals located in Hawai'i ( $V$. coralliilyticus OCN008 ${ }^{28}$ and V. coralliilyticus OCN014 ${ }^{27}$ ). Most recently, a fifth complete genome was sequenced from $V$. coralliilyticus isolated from oysters at a shellfish hatchery ( $V$. coralliilyticus $\mathrm{RE} 98^{26}$ ). We interrogated each genome for signatures of or complete prophage genomes.

Prophages in $\boldsymbol{V}$. coralliilyticus genomes. Our in silico analysis revealed the BAA450 genome contains an intact prophage genome that shares homology to the core region of the CTX $\varphi$ genome (Figs 1 and 2) and encodes a number of genes that share close identity to those encoded by a filamentous prophage, $\operatorname{VCY} \varphi$, isolated from an environmental $V$. cholerae population ${ }^{29}$ (Tables 2 and 3; Figs 2 and 3). The $\operatorname{VCY} \varphi$ prophage genome shares sequence similarity to the $\operatorname{CTX} \varphi$ prophage ${ }^{29}$, and like $\operatorname{CTX} \varphi$, is a novel virus that has evolved through HGT events and recombination. A prophage genome encoding a number of genes homologous to those encoded by $\operatorname{VCY} \varphi$ was also detected in the $V$. coralliilyticus $\mathrm{P} 1$ strain but is absent from the OCN008 and OCN014 strains from Hawai'i and the RE98 genome (Tables 2 and 3; Figs 1 and 2).

For the morphogenesis of the CTX $\varphi$ prophage, three genes known as zot (zonal occludens toxin), ace (accessory cholera enterotoxin) and cep (core-encoded cepin) are required ${ }^{30}$. Both the $z o t$ and ace genes are identified as minor coat proteins of the $\mathrm{CTX} \varphi$ prophage but also exhibit toxic activity by encoding for toxins that play a direct role in disrupting the intestines of humans with cholera disease ${ }^{30}$. A rst $\mathrm{B}$ gene (Figs 1a and 2) encodes a DNA-binding protein essential for prophage formation and is identified 

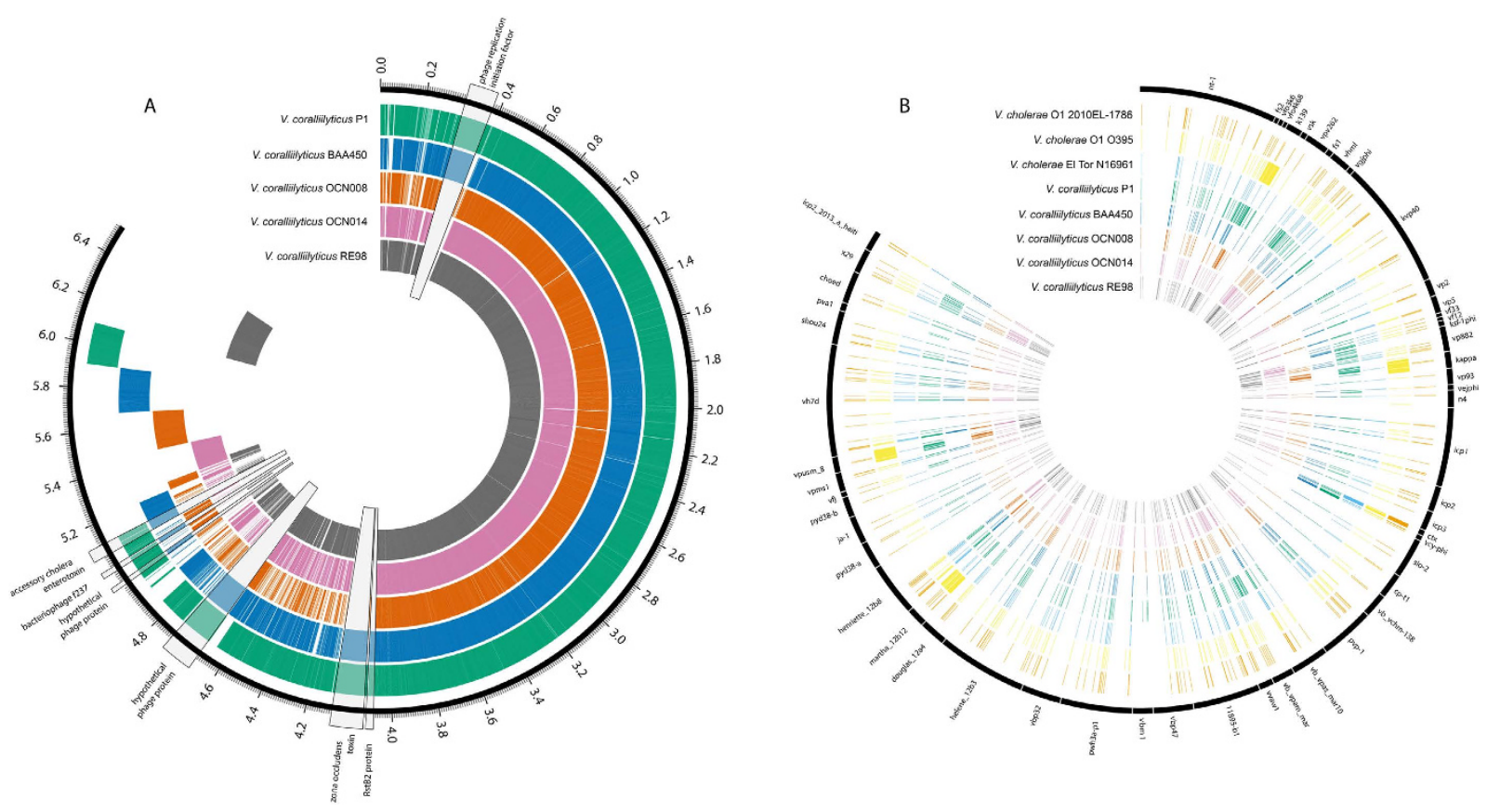

Figure 1. (a) Pangenome of the five Vibrio coralliilyticus strains. OrthoMCL v2.0.9 was used to generate clusters of similar proteins, which are indicated by overlapping regions. The tick marks indicate size of the pangenome in millions of base pairs (Mbps) and key prophage signatures are highlighted and magnified 100x. (b) Clustering of Vibrio coralliilyticus proteins and Vibrio cholerae proteins with known Vibrio phage proteins. OrthoMCL v2.0.9 was used to detect similarity between proteins in the V. coralliilyticus genomes and phage genomes, which are indicated by the overlapping tracks. To identify related protein clusters a minimum similarity of $50 \%$ and an e-value of $<10^{-5}$ was used. Overlapping lines denote the presence of a similar protein in each of the genomes. The genomes each have a unique colour and this is not related to similarity. The outer-most numbers in plot A only give a guide to the total size of the pan-genome; these do not represent locations within genomes. The orthoMCL program looks only for similar proteins with no regard to their location or synteny within different genomes and therefore, this plot should only be used to determine the presence or absence of particular proteins of interest.

in the СТX $\varphi$ genome as playing a role in the site-specific integration of the prophage into the host chromosome $^{31}$. Homologues of these genes are encoded by the genome of the Vibrio VCY $\varphi$-like prophage found in BAA450 and P1 (Table 3; Figs 1-3; Supplemental Figure 1). Identity scores for specific genes for these prophages in BAA450 and P1 are shown in Table 3. Sequence alignment of key genes in the BAA450 prophage genome with similar genes encoded by $\mathrm{VCY} \varphi$ genome is shown in Fig. 3. Genes in the BAA450 prophage that share close similarity with those in the $\mathrm{VCY} \varphi$ genome include a DNA replication initiation protein (identity $=66 \%$; score $=559$ ), a gene encoding a ssDNA-binding protein, similar to the $r s t \mathrm{~B}$ gene (identity $=53 \%$; score $=103$ ), a minor capsid protein gene similar to ace (identity $=29 \%$; score $=158)$ and a gene encoding a Zot-like protein (identity $=61 \%$; score $=578$ ) (Table 3 ).

An intact prophage was detected in the OCN014 genome that shares similarity to a plasmid-like prophage VP882 $2^{32}$ found in V. parahaemolyticus, a pathogen commonly associated with shellfish (Table 2; Figs $1 \mathrm{~b}$ and 2). This prophage genome shares similarity to a small number of genes in the Pseudomonas phage CTX $\varphi$-like genome found in P1 (Fig. 2). PHAST ${ }^{33}$ analysis of the OCN008 genome detected one intact prophage that shares similarity with a $V$. cholerae prophage $\mathrm{K} 139^{34}$ and also shares homology with the incomplete Kappa prophage located in the RE98 genome (Table 2; Figs 1b and 2). The phage K139 has been previously identified as associating with pathogenic $V$. cholerae strains but it is still unclear whether or how this prophage may increase pathogenic host fitness ${ }^{35}$. Strain RE98 has been identified as a particularly virulent pathogen responsible for high mortality rates in oyster shellfish hatcheries in the west coast of the US ${ }^{36}$. A second intact vB VpaM-like prophage was detected in the RE98 genome that shares homology to the Pseudomonas СTX $\varphi$-like prophage in P1 (Table 2: Figs $1 \mathrm{~b}$ and 2). A heat map analysis indicates similarity between the prophage genomes identified in this study (see Supplementary Fig. S1 online).

Pathogenicity islands in the $\boldsymbol{V}$. coralliilyticus genome. The synergistic effect of a number of genes, including toxicity genes introduced by $\operatorname{CTX} \varphi$, and a series of genes clustered in pathogenicity islands on the bacterial genome result in the evolution of $V$. cholerae ${ }^{7}$ to harmful epidemic and pandemic-causing 


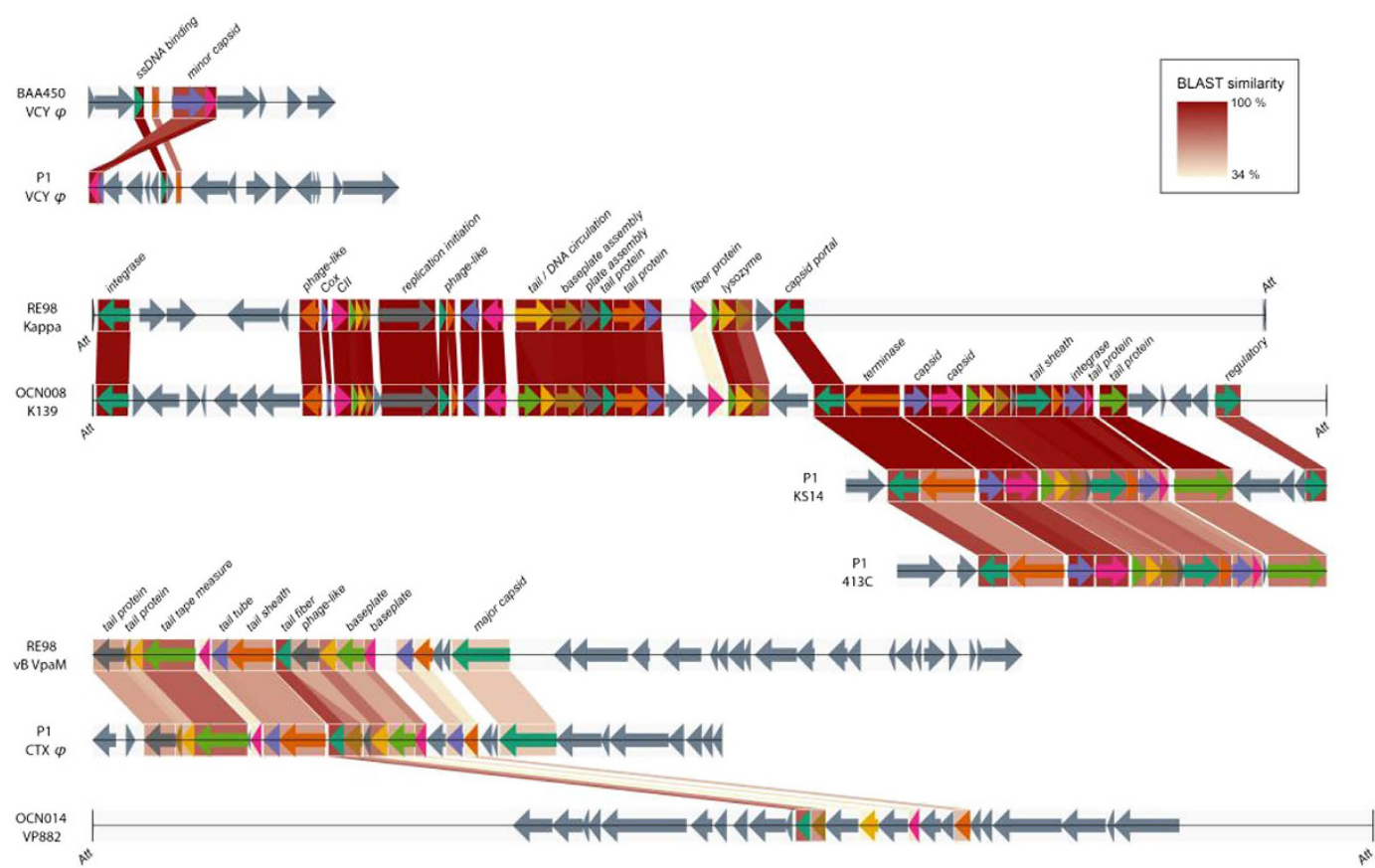

Figure 2. Genome organisation of prophages found in the genomes of five Vibrio coralliilyticus strains isolated from corals and oysters. BLAST values were determined using amino acid sequences with BLASTP. Linear ORF maps of the prophages were aligned based on their modular structures. ORFs or genes are represented by arrows oriented in the direction of transcription. Coloured arrows are annotated to indicate predicted function. Grey arrows represent unknown ORFs. The att (phage attachment sites), where identified, are indicated.

\begin{tabular}{|c|c|c|c|c|c|c|c|}
\hline VC strain & $\begin{array}{l}\text { No. of prophage } \\
\text { identified }\end{array}$ & $\begin{array}{l}\text { Prophage genome } \\
\text { length (kb) }\end{array}$ & $\begin{array}{l}\text { Prophages (closest similarity) } \\
\text { identified in VC genome }\end{array}$ & Score & Number of CDS & Region position & GC\% \\
\hline BAA450 & 1 & 7.8 & Intact $\mathrm{VCY} \varphi$ phage (NC_016162) & 95 & 9 & $1436462-1444336$ & 43.64 \\
\hline \multirow[t]{4}{*}{ P1 } & 4 & 15.3 & $\begin{array}{l}\text { Incomplete Burkholderia- like } \\
\text { phage KS14 (NC_015273) }\end{array}$ & 60 & 18 & $511097-526448$ & 40.42 \\
\hline & & 20 & $\begin{array}{l}\text { Intact Pseudomonas } \varphi \text { CTX phage } \\
\text { (NC_003278) }\end{array}$ & 140 & 29 & $1856473-1876563$ & 45.13 \\
\hline & & 13.7 & $\begin{array}{l}\text { Incomplete Yersinia phage } \\
\text { L-413C (NC_004745) }\end{array}$ & 60 & 16 & $2505529-2519246$ & 45.16 \\
\hline & & 9.9 & $\begin{array}{l}\text { Incomplete VCY } \varphi \text { phage } \\
\text { (NC_016162) }\end{array}$ & 20 & 16 & $5482028-5491938$ & 46.0 \\
\hline OCN008 & 1 & 39.3 & $\begin{array}{l}\text { Intact Vibrio phage K139 } \\
\text { (NC_003313) }\end{array}$ & 130 & 52 & $1044704-1084092$ & 44.12 \\
\hline OCN014 & 1 & 40.8 & $\begin{array}{l}\text { Intact Vibrio phage VP882 } \\
\text { (NC_009016) }\end{array}$ & 140 & 22 & $1698322-1739200$ & 44.66 \\
\hline \multirow[t]{2}{*}{ RE98 } & 2 & 37.3 & $\begin{array}{c}\text { Incomplete Vibrio Kappa phage } \\
\text { (NC_003313) }\end{array}$ & 90 & 28 & $653256-690638$ & 44.12 \\
\hline & & 13.3 & $\begin{array}{c}\text { Intact Vibrio vB VpaM phage } \\
\text { (NC_019722) }\end{array}$ & 130 & 17 & $934714-948029$ & 46.28 \\
\hline
\end{tabular}

Table 2. Details of prophages identified in five publically available Vibrio corallilyticus (VC) genomes.

strains. The pathogenicity islands (namely Vibrio Pathogenicity Island I and II, VPI-I/II) consist of virulence genes, a transposase gene, specific (att-like) attachment sites flanking each end of the island, and an integrase with homology to a prophage integrase gene ${ }^{7}$. The TCP gene cluster induces virulent Vibrios to secrete the exotoxin cholera toxin (CTX) and is encoded on a pathogenicity island within the Vibrio chromosome ${ }^{7}$. These genes are present in BAA450 and P1, suggesting these elements may influence the virulence of BAA450 and P1. Both BAA450 and P1 genomes carry a Coralliilyticus Pathogenicity Island-I (CPI-I) that is located at a chromosomal site similar to the dif-like region that accommodates an insertion site for the prophages $\mathrm{CTX} \varphi$ and $\mathrm{f} 237$ of $V$. cholerae $^{6}$ and $V$. parahaemolyticus ${ }^{37}$, respectively. 


\begin{tabular}{|l|c|c|c|c|c|c|}
\hline VC strain CDS position & VCY $\varphi$ Accession No. & Gene identified & Identity (\%) & E-value & Score & Query coverage (\%) \\
\hline BAA450_1 & YP_004934223.1 & Hypothetical protein & 43 & $1 \mathrm{e}-13$ & 66 & 81 \\
\hline BAA450_2 & YP_004934224.1 & DNA replication initiation protein & 66 & 0 & 559 & 98 \\
\hline BAA450_3 & YP_004934225.1 & ssDNA-binding protein (RstB) & 53 & $9 \mathrm{e}-28$ & 103 & 88 \\
\hline BAA450_4 & YP_004934227.1 & Hypothetical protein & 47 & $2 \mathrm{e}-10$ & 57 & 100 \\
\hline BAA450_5 & YP_004934228.1 & Minor capsid protein & 29 & $5 \mathrm{e}-41$ & 158 & 99 \\
\hline BAA450_6 & YP_004934229.1 & Hypothetical protein & 58 & $3 \mathrm{e}-41$ & 139 & 99 \\
\hline BAA450_7 & YP_004934230.1 & ZOT-like protein & 61 & 0 & 578 & 96 \\
\hline P1_1 & YP_004934229.1 & Hypothetical protein & 47 & $4 \mathrm{e}-17$ & 75.5 & 100 \\
\hline P1_2 & YP_004934225.1 & Minor capsid protein & 43 & $4 \mathrm{e}-06$ & 48 & 85 \\
\hline P1_7 & YP_004934225.1 & ssDNA-binding protein (RstB) & 52 & $4 \mathrm{e}-09$ & 53.5 & 89 \\
\hline P1_8 & YP_004934226.1 & Hypothetical protein & 54 & 2e-17 & 75.5 & 100 \\
\hline P1_9 & YP_004934227.1 & Hypothetical protein & 52 & $5 \mathrm{e}-07$ & 47 & 94 \\
\hline
\end{tabular}

Table 3. Similarity values for ORFs in V. corallilyticus (VC) strains BAA450 and P1 genomes with closest matches to $\operatorname{VCY} \varphi$ phage genes.

The TCP is encoded for in CPI-1 of BAA450 and P1. While BAA450 has a similar genome arrangement to $V$. cholerae with the presence of a second pathogenicity island (CPI-II) ${ }^{20}$, the P1 genome does not contain a second pathogenicity island CPI-II. It is noteworthy that the CPI-II in BAA450 bears similarity to a second pathogenicity island found in the genome of the seventh pandemic strain of $V$. cholerae ${ }^{7}$. Further, of the five genomes analysed here, the BAA450, P1, OCN014 and RE98 genomes encode for a RTX (repeats in toxin) protein that is a harmful pore-forming toxin required for human host colonisation in incidences of infection by $V$. cholerae $^{30}$. The RTX gene cluster in $V$. cholerae is physically located close to the CTX $\varphi$ genome ${ }^{38}$. A significant upregulation of the RTX protein and a number of proteins located on the same pathogenicity island as the RTX protein occurs in BAA450 at increased temperatures of $27^{\circ} \mathrm{C}$ and above, contributing to increased virulence at higher temperatures ${ }^{20}$.

\section{Discussion}

Prophage infections can transform ordinary bacteria to virulent pathogens, shown in a wide range of species including Vibrio cholerae ${ }^{6}$, Staphylococcus aureus, Clostridium botulinum, Corynebacterium diphtheriae, Streptococcus pyrogenes and Escherichia coli ${ }^{8,39}$. A landmark discovery in bacteriophage research revealed the role of lysogenic prophages in the conversion of the bacterium Vibrio cholerae from a non-pathogenic to a virulent strain, resulting in often devastating cholera outbreaks among human populations ${ }^{4-6}$. Our analysis shows that the same scenario may exist for the coral pathogen, Vibrio coralliilyticus, and future studies should rigorously address this previously overlooked possibility.

BAA450 was reported over a decade ago as causing bleaching and tissue necrosis in the coral species Pocillopora damicornis ${ }^{14,17}$. This strain was seen to induce bleaching at temperatures between $24-26^{\circ} \mathrm{C}$, but not at lower temperatures. An increase in protease activity resulting in the release of high amounts of toxic extracellular proteases was observed at temperatures between $27-29^{\circ} \mathrm{C}$, which resulted in mortality of the coral ${ }^{17}$. The presence of an intact prophage genome in the BAA450 genome and an incomplete prophage in P1 that share similarities to the genome of $\mathrm{CTX} \varphi$ prophage, responsible for toxigenic conversion of $V$. cholerae strains, suggests that similar prophage-driven virulence mechanisms could operate in both the human and coral system. Extracellular zinc-metalloproteases expressed by $V$. coralliilyticus strain P1 were previously reported to be the driver of tissue damage in WS infections ${ }^{17,24}$ but these enzymes were not the only drivers as demonstrated by subsequent mutant infection experiments ${ }^{25}$. These studies did not include an assessment of the presence of СTX $\varphi$ prophage-derived toxins, such as ZOT and ACE proteins. We therefore encourage future experiments towards assessing the expression of these virulence factors in infections by $V$. coralliilyticus strains.

The OCN008 strain has been indicated as an aetiological agent in white syndrome of the coral species Montipora capitata ${ }^{28}$. Unlike strains BAA450 and P1, the pathogenesis of OCN008 is reported as being temperature-independent ${ }^{28}$ with only a relatively slower onset of infection observed at lower temperatures, resulting in tissue loss without bleaching. Previous comparative analysis of the genome sequences of BAA450, P1 and OCN008 reported approximately 84\% similarity between the genomes of OCN008 and BAA450 and 89\% similarity between the OCN008 and P1 genomes ${ }^{28}$. Genes upregulated at least 4 -fold in BAA450 by a rise in temperature shared $97-100 \%$ sequence identity with genes in OCN $008^{28}$. Why does infection by OCN008 appear to be independent of increasing temperature? The intact prophage genome detected in the OCN008 genome shares similarity to the Vibrio prophage K139 that is also present in the $V$. cholerae $\mathrm{O} 1 \mathrm{O} 395$ genome (Fig. 2). It is possible that the presence of this intact prophage in the OCN008 genome and the absence of a CTX $\varphi$-like prophage genome results in 


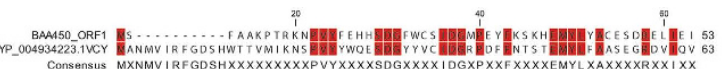

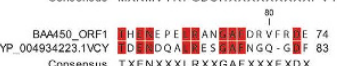

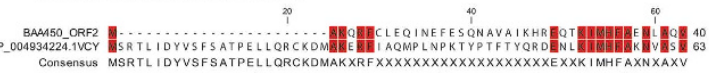

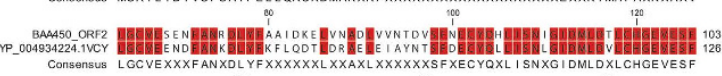

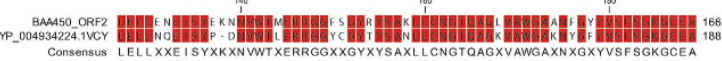

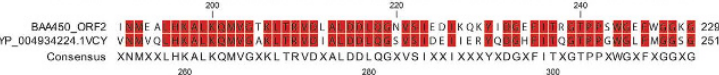

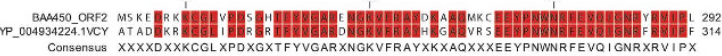

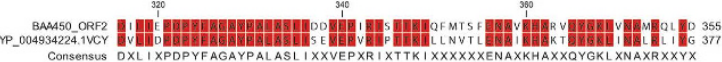

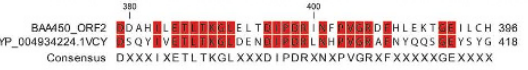

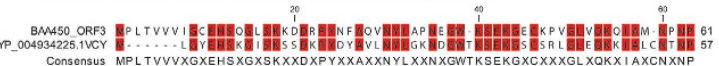

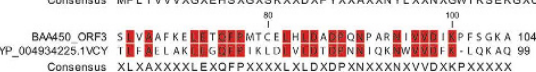

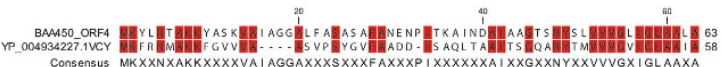

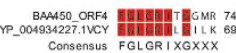

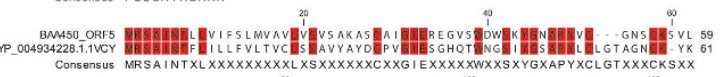

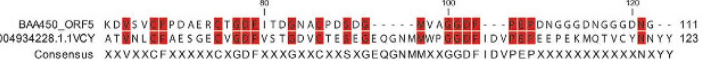

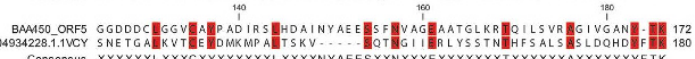

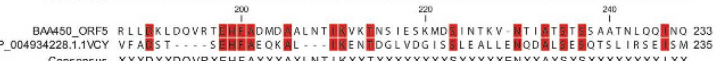

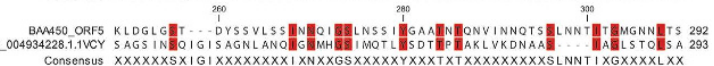

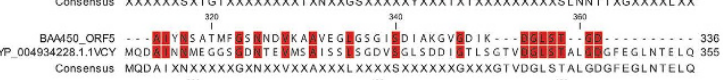

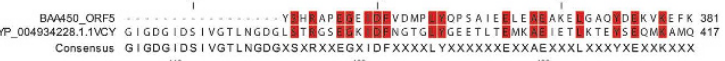

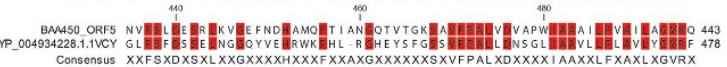

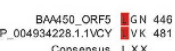

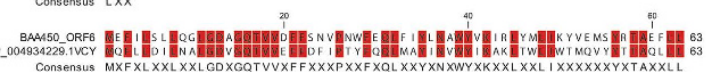

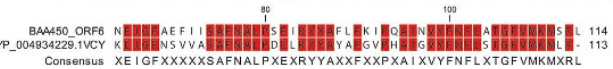

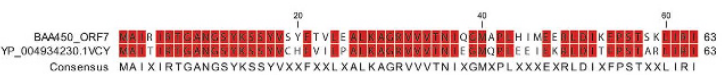

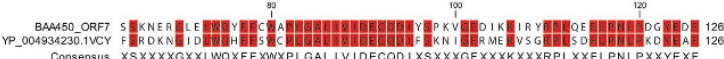

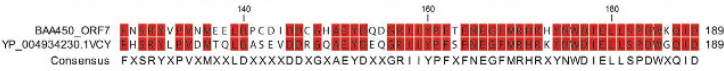

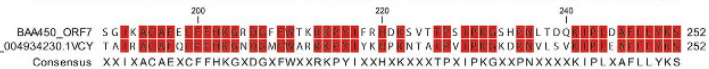

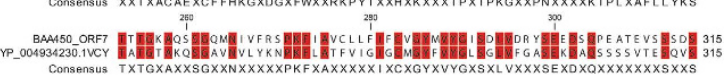

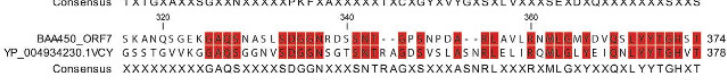

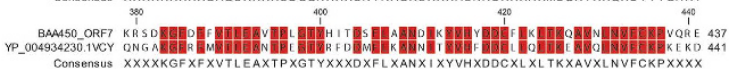

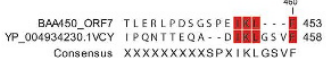

Figure 3. Amino-acid alignment of 7 of 9 ORFs encoded by the BAA450 prophage with 7 of 11 ORFs in the Vibrio phage VCY-phi (Accession No. NC_016162.1). Conserved residues are shaded in red. Dashes indicate gaps. 
a different disease profile compared to those of the BAA450 and P1 temperature-dependent infections. OCN014 has been isolated from corals exhibiting symptoms of $\mathrm{WS}^{27}$. Further investigations are needed to link the presence of OCN014 to coral disease and we encourage the inclusion of virus characterisation in such experimental work.

$V$. cholerae is autochthonous to coastal and estuarine waters and rising seawater temperatures have been indicated as a major influence on $V$. cholerae pandemics ${ }^{40}$. Does the presence of a prophage similar to the $\mathrm{CTX} \varphi$ prophage in the BAA450 and P1 genomes explain these strain's increased virulence with rising temperature ${ }^{14,17,41,42}$ ? It has been predicted, based on time-series studies, that as global temperatures rise, cholera outbreaks will increase ${ }^{40,43,44}$. Comparably, researchers have indicated the role of seasonal dynamics, microbial interactions and environmental factors driving the occurrence of disease caused by $V$. coralliilyticus and these are predicted to increase with ongoing environmental change ${ }^{16,41,45}$. For instance, a recent study reported $V$. coralliilyticus populations in $P$. damicornis increased by as much as four times with elevated seawater temperatures ${ }^{46}$. We postulate that as temperatures increase the BAA450 and $\mathrm{P} 1$ prophages will undergo increased active replication along with host genome replication, and hence, their associated virulence factors (e.g. ZOT and ACE proteins, as well as TCP and RTX) will increase. With a changing climate, sea surface temperatures have steadily risen accompanied by expanding coral pathogen ranges ${ }^{47}$.

There have been greater than 30 coral diseases described ${ }^{41,48}$ but only a few diseases have been attributed to causative bacterial pathogens, namely Thalassotalea loyana (formerly Thalassomonas loyana) in white plague disease ${ }^{49}$, Vibrio shiloi in bleaching of the coral Oculina patagonica ${ }^{16,50}$ and V. coralliilyticus in coral bleaching and white syndrome disease $\mathrm{e}^{14,15,17}$. Coral disease profiling is complex and suspected pathogens of disease have been identified in apparently healthy corals ${ }^{41,51}$. The aetiology and pathogenicity of $V$. coralliilyticus has generated much interest, but very little is understood about the dynamics of infection. The parallels between the Vibrio cholerae paradigm and an equivalent scenario in corals is highly relevant and comparable, as disease in both human and coral populations are influenced by environmental factors that are being altered through anthropogenic influences and changes in climatic conditions.

We encourage further experimental studies into the role of viruses in Vibrio infections in corals. Previous studies have described the presence of viruses associated with coral diseases ${ }^{52-54}$ but none have investigated the role of prophages and lysogenic conversion. As all $V$. coralliilyticus genomes sequenced to date have been isolated from corals with disease/bleaching symptoms, at present we cannot make comparisons and form conclusions on the presence of these prophages in genomes of $V$. coralliilyticus isolated from disease-free samples. We anticipate future sequencing efforts to include genomes of $V$. coralliilyticus from healthy corals, or other such sources, to enable an in-depth comparison. In this present study, we indicate possible signatures to target in $V$. coralliilyticus to screen for prophages. We envisage our in silico analysis to be a foundation and starting point for future experimental research on the role of lysogenic conversion in pathogenicity of Vibrio and the environmental conditions that potentially trigger virulence. Coral disease is not caused by a single factor but is the result of a complex interplay among biotic and abiotic factors and prophages are a potential central driver of virulence.

\section{Methods}

For interrogative and comparative analysis, whole genome sequences of five published $V$. coralliilyticus genomes were downloaded from the NCBI database, March 2015. The V. coralliilyticus strain names and their accession numbers are as follows and summarised in Table 1: V. coralliilyticus ATCC-BAA-450 ${ }^{14,20}$ accession number ACZN00000000.1; V. coralliilyticus $\mathrm{P} 1^{15,25}$ accession number AEQS00000000.1; V. coralliilyticus OCN008 ${ }^{28}$ accession number AVOO00000000.1; V. coralliilyticus OCN014 ${ }^{27}$ accession numbers CP009264 and CP009265; and V. coralliilyticus RE98 ${ }^{26}$ accession numbers CP009617 and CP009618 both OCN014 and RE98 genomes have been deposited under BioProject PRJNA224116. The five $V$. coralliilyticus genome sequences were curated and analysed using Ortho MCL v2.0.95 , an analysis tool that identifies orthologous groups for use in comparative genomics and evolutionary processes. Three $V$. cholerae representative reference genomes were downloaded from NCBI: V. cholerae O1 2010EL-1786 ${ }^{56}$ GenBank accession number CP003069 and CP003070; V. cholerae O1 O395 ${ }^{57}$ GenBank accession number CP000626 and CP000627; V. cholerae El Tor N16961 ${ }^{58}$ GenBank accession number AE003852 and AE003853 and were also included in an OrthoMCL analysis. Comparative genomic analysis was visualised using the software tool $\operatorname{Circos}^{59}$ version 0.66. To detect integrated prophage genomes a PHAST (Prophage Search Tool, updated 01/05/2015) $)^{33}$ analysis was conducted on the five V. coralliilyticus genomes.

\section{References}

1. Reen, F. J., Almagro-Moreno, S., Ussery, D. \& Boyd, E. F. The genomic code: inferring Vibrionaceae niche specialization. Nat. Rev. Microbiol. 4, 697-704 (2006).

2. Dziejman, M. et al. Genomic characterization of non-O1, non-O139 Vibrio cholerae reveals genes for a type III secretion system. Proc. Nat. Acad. Sci. USA 102, 3465-3470, doi: 10.1073/pnas.0409918102 (2005).

3. Gennari, M., Ghidini, V., Caburlotto, G. \& Lleo, M. M. Virulence Genes and Pathogenicity Islands in Environmental Vibrio Strains Nonpathogenic to Humans. Vol. 82 (2012).

4. Levin, B. R. \& Tauxe, R. V. Cholera: Nice bacteria and bad viruses. Curr. Biol. 6, 1389-1391, doi: 10.1016/s0960-9822(96)007385 (1996). 
5. McLeod, S. M., Kimsey, H. H., Davis, B. M. \& Waldor, M. K. CTX phi and Vibrio cholerae: exploring a newly recognized type of phage-host cell relationship. Mol. Microbiol. 57, 347-356, doi: 10.1111/j.1365-2958.04676.x (2005).

6. Waldor, M. K. \& Mekalanos, J. J. Lysogenic conversion by a filamentous phage encoding cholera toxin. Science 272, 1910-1914, doi: $10.1126 /$ science.272.5270.1910 (1996).

7. Faruque, S. M. \& Mekalanos, J. J. Pathogenicity islands and phages in Vibrio cholerae evolution. Trends Microbiol. 11, 505-510, doi: doi: 10.1016/j.tim.2003.09.003 (2003).

8. Brüssow, H., Canchaya, C. \& Hardt, W.-D. Phages and the evolution of bacterial pathogens: from genomic rearrangements to lysogenic conversion. Microbiol. Mol. Biol. Rev. 68, 560-602, doi: 10.1128/mmbr.68.3.560-602.2004 (2004).

9. Wilkinson, C. Status of Coral Reefs of the World: 2008. pp 1-304. (Global Coral Reef Monitoring Network and Reef and Rainforest Research Centre, 2008).

10. Hoegh-Guldberg, O. Climate change, coral bleaching and the future of the world's coral reefs. Mar. Freshw. Res. 50, 839-866, doi: $10.1071 / \mathrm{mf} 99078(1999)$.

11. Harvell, C. D. et al. Review: Marine ecology - Emerging marine diseases - Climate links and anthropogenic factors. Science 285, 1505-1510, doi: 10.1126/science.285.5433.1505 (1999).

12. Bruno, J. F. \& Selig, E. R. Regional decline of coral cover in the Indo-Pacific: Timing, extent, and subregional comparisons. PLoS ONE 2, e711, doi: 10.1371/journal.pone.0000711 (2007).

13. Willis, B. E., Page, C. A. \& Dinsdale, E. A. in Coral Health and Disease. 69-104 (Springer-Verlag Publishing, 2004).

14. Ben-Haim, Y. et al. Vibrio coralliilyticus sp nov., a temperature-dependent pathogen of the coral Pocillopora damicornis. Int. J. Syst. Evol. Microbiol. 53, 309-315, doi: 10.1099/ijs.0.02402-0 (2003).

15. Sussman, M., Willis, B. L., Victor, S. \& Bourne, D. G. Coral pathogens identified for white syndrome (WS) epizootics in the Indo-Pacific. PLoS ONE 3, e2393, doi: 10.1371/journal.pone.0002393 (2008).

16. Mills, E., Shechtman, K., Loya, Y. \& Rosenberg, E. Bacteria appear to play important roles in both causing and preventing the bleaching of the coral Oculina patagonica. Mar. Ecol. Prog. Ser. 489, 155-162, doi: 10.3354/meps10391 (2013).

17. Ben-Haim, Y., Zicherman-Keren, M. \& Rosenberg, E. Temperature-regulated bleaching and lysis of the coral Pocillopora damicornis by the novel pathogen Vibrio coralliilyticus. Appl. Environ. Microbiol. 69, 4236-4242, doi: 10.1128/aem.69.7.42364242.2003 (2003).

18. Brown, B. E. Coral bleaching: causes and consequences. Coral Reefs 16, S129-S138, doi: 10.1007/s003380050249 (1997).

19. Roff, G. et al. The ecology of 'Acroporid white syndrome', a coral disease from the southern Great Barrier Reef. PLoS ONE 6, e26829, doi: 10.1371/journal.pone.0026829 (2011).

20. Kimes, N. E. et al. Temperature regulation of virulence factors in the pathogen Vibrio coralliilyticus. ISMEJ 6, 835-846, doi: 10.1038/ismej.2011.154 (2012).

21. Vidal-Dupiol, J. et al. Innate immune responses of a scleractinian coral to vibriosis. J. Biol. Chem. 286, 22688-22698, doi: 10.1074/jbc.M110.216358 (2011)

22. Ainsworth, T., Fine, M., Roff, G. \& Hoegh-Guldberg, O. Bacteria are not the primary cause of bleaching in the Mediterranean coral Oculina patagonica. ISMEJ 2, 67-73, doi: 10.1038/ismej.2007.88 (2008).

23. Kushmaro, A., Banin, E., Loya, Y., Stackebrandt, E. \& Rosenberg, E. Vibrio shiloi sp nov., the causative agent of bleaching of the coral Oculina patagonica. Int. J. Syst. Evol. Microbiol. 51, 1383-1388 (2001).

24. Sussman, M. et al. Vibrio zinc-metalloprotease causes photoinactivation of coral endosymbionts and coral tissue lesions. PLoS ONE 4, doi: 10.1371/journal.pone.0004511 (2009).

25. Santos, E. d. O. et al. Genomic and proteomic analyses of the coral pathogen Vibrio coralliilyticus reveal a diverse virulence repertoire. ISMEJ 5, 1471-1483, doi: 10.1038/ismej.2011.19 (2011).

26. Richards, G. P., Bono, J. L., Watson, M. A. \& Needleman, D. S. Complete genome sequence for the shellfish pathogen Vibrio coralliilyticus RE98 isolated from a shellfish hatchery. Genome Announc 2, e01253-01214, doi: 10.1128/genomeA.01253-14 (2014).

27. Ushijima, B. et al. Complete genome sequence of Vibrio coralliilyticus strain OCN014, isolated from a diseased coral at Palmyra Atoll. Genome Announc 2, e01318-14, doi: 10.1128/genomeA.01318-14 (2014).

28. Ushijima, B. et al. Vibrio coralliilyticus strain OCN008 is an etiological agent of acute Montipora shite syndrome. Appl. Environ. Microbiol. 80, 2102-2109, doi: 10.1128/aem.03463-13 (2014).

29. Xue, H., Xu, Y., Boucher, Y. \& Polz, M. F. High frequency of a novel filamentous phage, VCY $\varphi$, within an environmental Vibrio cholerae population. Appl. Environ. Microbiol. 78, 28-33, doi: 10.1128/aem.06297-11 (2012).

30. Chaudhuri, K. \& Chatterjee, S. N. Cholera Toxins., 321 (Springer, 2009).

31. Falero, A. et al. DNA binding proteins of the filamentous phages CTX $\varphi$ and VGJ $\varphi$ of Vibrio cholerae. J. Bacteriol. 191, 5873-5876, doi: 10.1128/jb.01206-08 (2009).

32. Lan, S.-F. et al. Characterization of a new plasmid-Like prophage in a pandemic Vibrio parahaemolyticus O3:K6 strain. Appl. Environ. Microbiol. 75, 2659-2667, doi: 10.1128/aem.02483-08 (2009).

33. Zhou, Y., Liang, Y., Lynch, K. H., Dennis, J. J. \& Wishart, D. S. PHAST: A fast phage search tool. Nucleic Acids Res., doi: 10.1093/ nar/gkr485 (2011).

34. Kapfhammer, D., Blass, J., Evers, S. \& Reidl, J. Vibrio cholerae phage K139: complete genome sequence and comparative genomics of related phages. J. Bacteriol. 184, 6592-6601, doi: 10.1128/jb.184.23.6592-6601.2002 (2002).

35. Nesper, J., Blaß, J., Fountoulakis, M. \& Reidl, J. Characterization of the major control region of Vibrio cholerae bacteriophage K139: Immunity, exclusion, and integration. J. Bacteriol. 181, 2902-2913 (1999).

36. Richards, G. P., Watson, M. A., Needleman, D. S., Church, K. M. \& Häse, C. C. Mortalities of Eastern and Pacific oyster larvae caused by the pathogens Vibrio coralliilyticus and Vibrio tubiashii. Appl. Environ. Microbiol. 81, 292-297, doi: 10.1128/aem.0293014 (2015).

37. Nasu, H. et al. A filamentous phage associated with recent pandemic Vibrio parahaemolyticus O3:K6 strains. J. Clin. Microbiol. 38, 2156-2161 (2000).

38. Lin, W. et al. Identification of a Vibrio cholerae RTX toxin gene cluster that is tightly linked to the cholera toxin prophage. Proc. Nat. Acad. Sci. USA 96, 1071-1076, doi: 10.1073/pnas.96.3.1071 (1999).

39. Fortier, L.-C. \& Sekulovic, O. Importance of prophages to evolution and virulence of bacterial pathogens. Virulence 4, 354-365, doi: 10.4161/viru.24498 (2013)

40. Colwell, R. R. Global climate and infectious disease: The cholera paradigm. Science 274, 2025-2031, doi: 10.1126/ science.274.5295.2025 (1996)

41. Bourne, D. G. et al. Microbial disease and the coral holobiont. Trends Microbiol. 17, 554-562, doi: doi: 10.1016/j.tim.2009.09.004 (2009).

42. Bruno, J. F. et al. Thermal Stress and Coral Cover as Drivers of Coral Disease Outbreaks. PLoS Biol 5, e124, doi: 10.1371/journal. pbio.0050124 (2007).

43. Constantin de Magny, G. et al. Environmental signatures associated with cholera epidemics. Proc. Nat. Acad. Sci. USA 105, 17676-17681, doi: 10.1073/pnas.0809654105 (2008). 
44. Reyburn, R. et al. Climate variability and the outbreaks of cholera in Zanzibar, East Africa: A time series analysis. Am. J. Trop. Med. Hyg. 84, 862-869, doi: 10.4269/ajtmh.2011.10-0277 (2011).

45. Ainsworth, T. D. \& Hoegh-Guldberg, O. Bacterial communities closely associated with coral tissues vary under experimental and natural reef conditions and thermal stress. Aquat. Biol. 4, 289-296, doi: 10.3354/ab00102 (2009).

46. Tout, J. et al. Increased seawater temperature increases the abundance and alters the structure of natural Vibrio populations associated with the coral Pocillopora damicornis. Front. Microbiol. 6, doi: 10.3389/fmicb.2015.00432 (2015).

47. Harvell, D. et al. Coral disease, environmental drivers and the balance between coral and microbial associates. Oceanography 20, 172-195 (2007).

48. Sheridan, C., Kramarsky-Winter, E., Sweet, M., Kushmaro, A. \& Leal, M. C. Diseases in coral aquaculture: causes, implications and preventions. Aquaculture 396, 124-135, doi: 10.1016/j.aquaculture.2013.02.037 (2013).

49. Thompson, F. L. et al. Thalassomonas loyana sp nov, a causative agent of the white plague-like disease of corals on the Eilat coral reef. Int. J. Syst. Evol. Microbiol. 56, 365-368, doi: 10.1099/ijs.0.63800-0 (2006).

50. Rubio-Portillo, E., Yarza, P., Penalver, C., Ramos-Espla, A. A. \& Anton, J. New insights into Oculina patagonica coral diseases and their associated Vibrio spp. communities. ISMEJ 8, 1794-1807, doi: 10.1038/ismej.2014.33 (2014).

51. Ritchie, K. B. Regulation of microbial populations by coral surface mucus and mucus-associated bacteria. Mar. Ecol. Prog. Ser. 322, 1-14, doi: 10.3354/meps322001 (2006).

52. Lawrence, S., Davy, J., Aeby, G., Wilson, W. \& Davy, S. Quantification of virus-like particles suggests viral infection in corals affected by Porites tissue loss. Coral Reefs 33, 687-691, doi: 10.1007/s00338-014-1168-8 (2014).

53. Pollock, F. J. et al. Abundance and morphology of virus-like particles associated with the coral Acropora hyacinthus differ between healthy and white syndrome-infected states. Mar. Ecol. Prog. Ser. 510, 39-43, doi: 10.3354/meps10927 (2014).

54. Soffer, N., Brandt, M. E., Correa, A. M. S., Smith, T. B. \& Thurber, R. V. Potential role of viruses in white plague coral disease. ISMEJ 8, 271-283, doi: 10.1038/ismej.2013.137 (2014).

55. Li, L., Stoeckert, C. J. \& Roos, D. S. OrthoMCL: Identification of ortholog groups for eukaryotic genomes. Genome Res. 13, 2178-2189, doi: $10.1101 /$ gr.1224503 (2003)

56. Reimer, A. R. et al. Comparative genomics of Vibrio cholerae from Haiti, Asia, and Africa. Emerging Infect. Dis. 17, 2113-2121, doi: 10.3201/eid1711.110794 (2011)

57. Feng, L. et al. A recalibrated molecular clock and independent origins for the cholera pandemic clones. PLoS ONE 3, e4053, doi: 10.1371/journal.pone.0004053 (2008).

58. Heidelberg, J. F. et al. DNA sequence of both chromosomes of the cholera pathogen Vibrio cholerae. Nature 406, 477-483, doi: $10.1038 / 35020000(2000)$.

59. Krzywinski, M. I. et al. Circos: An information aesthetic for comparative genomics. Genome Res. 19, 1639-1645, doi: 10.1101/ gr.092759.109 (2009).

\section{Acknowledgements}

We acknowledge funding from the Australian Research Council (Future Fellowship \#FT100100088 to M.v.O.) and from the Australian Institute of Marine Science.

\section{Author Contributions}

K.D.W. conceived the study. K.D.W., M.J.N. and P.B. performed data analysis. M.J.N. and K.D.W. prepared the figures. K.D.W., C.R.V., P.B. and M.v.O. wrote the paper. All authors reviewed the manuscript.

\section{Additional Information}

Supplementary information accompanies this paper at http://www.nature.com/srep

Competing financial interests: The authors declare no competing financial interests.

How to cite this article: Weynberg, K. D. et al. From cholera to corals: Viruses as drivers of virulence in a major coral bacterial pathogen. Sci. Rep. 5, 17889; doi: 10.1038/srep17889 (2015).

(c) (i) This work is licensed under a Creative Commons Attribution 4.0 International License. The images or other third party material in this article are included in the article's Creative Commons license, unless indicated otherwise in the credit line; if the material is not included under the Creative Commons license, users will need to obtain permission from the license holder to reproduce the material. To view a copy of this license, visit http://creativecommons.org/licenses/by/4.0/ 